\title{
On the Lebesgue constant of barycentric rational Hermite interpolants at equidistant nodes
}

\author{
Emiliano Cirillo · Kai Hormann
}

\begin{abstract}
Barycentric rational Floater-Hormann interpolants compare favourably to classical polynomial interpolants in the case of equidistant nodes, because the Lebesgue constant associated with these interpolants grows logarithmically in this setting, in contrast to the exponential growth experienced by polynomials. In the Hermite setting, in which also the first derivatives of the interpolant are prescribed at the nodes, the same exponential growth has been proven for polynomial interpolants, and the main goal of this paper is to show that much better results can be obtained with a recent generalization of Floater-Hormann interpolants. After summarizing the construction of these barycentric rational Hermite interpolants, we study the behaviour of the corresponding Lebesgue constant and prove that it is bounded from above by a constant. Several numerical examples confirm this result.
\end{abstract}

\section{Citation Info}

Journal

Journal of Computational and Applied Mathematics

Volume

349, March 2019

Pages

292-301

\section{Introduction}

Let $n \in \mathbb{N}$ and $X_{n}=\left\{x_{0}, x_{1}, \ldots, x_{n}\right\}$ be a set of $n+1$ nodes, such that $0=x_{0}<x_{1}<\cdots<x_{n}=1$. Given two sets of basis functions $b_{0, i}$ and $b_{1, i}, i=0, \ldots, n$, satisfying

$$
\begin{array}{ll}
b_{0, i}\left(x_{j}\right)=\delta_{i, j}, & b_{0, i}^{\prime}\left(x_{j}\right)=0, \\
b_{1, i}\left(x_{j}\right)=0, & b_{1, i}^{\prime}\left(x_{j}\right)=\delta_{i, j},
\end{array}
$$

for $j=0, \ldots, n$, and some $f \in C^{1}[0,1]$, we are interested in the class of linear Hermite interpolants $h_{n}:[0,1] \rightarrow \mathbb{R}$ of $f$ of the form

$$
h_{n}(x)=\sum_{i=0}^{n} b_{0, i}(x) f_{i}+\sum_{i=0}^{n} b_{1, i}(x) f_{i}^{\prime}
$$

where

$$
f_{i}=f\left(x_{i}\right), \quad f_{i}^{\prime}=f^{\prime}\left(x_{i}\right) .
$$

An example is given by the classical polynomial Hermite interpolants [5, 9], where the basis functions are defined as

$$
b_{0, i}(x)=\left(1-2\left(x-x_{i}\right) \ell_{i}^{\prime}\left(x_{i}\right)\right) \ell_{i}(x)^{2}, \quad b_{1, i}(x)=\left(x-x_{i}\right) \ell_{i}(x)^{2},
$$

with $\ell_{i}$ denoting the usual Lagrange basis polynomials

$$
\ell_{i}(x)=\prod_{\substack{j=0 \\ j \neq i}}^{n} \frac{x-x_{j}}{x_{i}-x_{j}} .
$$

Extending the concept of the Lebesgue constant from Lagrange to Hermite interpolation, we call

$$
\Lambda_{n}=\sup _{\|f\|_{1}=1}\left\|h_{n}\right\|
$$

the Lebesgue constant of the Hermite interpolant (1), where $\|\cdot\|$ is the supremum norm in $C[0,1]$ and

$$
\|f\|_{1}=\|f\|+\left\|f^{\prime}\right\| .
$$

It is then easy to verify [6] that

$$
\Omega_{1, n} \leq \Lambda_{n} \leq \max \left\{\Omega_{0, n}, \Omega_{1, n}\right\}
$$


where

$$
\Omega_{0, n}=\max _{0 \leq x \leq 1} \Omega_{0, n}(x), \quad \Omega_{1, n}=\max _{0 \leq x \leq 1} \Omega_{1, n}(x)
$$

and

$$
\Omega_{0, n}(x)=\sum_{i=0}^{n}\left|b_{0, i}(x)\right|, \quad \Omega_{1, n}(x)=\sum_{i=0}^{n}\left|b_{1, i}(x)\right| .
$$

Both $\Omega_{0, n}$ and $\Omega_{1, n}$ play a crucial role in measuring the approximation quality of $h_{n}$, since [7]

$$
\left\|f-h_{n}\right\| \leq\left(1+\Omega_{0, n}+\Omega_{1, n}\right)\left\|f^{\prime}-p_{2 n}\right\|,
$$

where $p_{2 n}$ is the polynomial of degree at most $2 n$ that approximates $f^{\prime}$ best on $[0,1]$, and have therefore been investigated intensively. For example, it is known that

$$
\Omega_{0, n}=1, \quad \Omega_{1, n} \leq \frac{1}{\rho},
$$

for $\rho$-normal sets of nodes [7], which implies convergence of the Hermite interpolation process. Examples of such sets are given by the roots of the Jacobi polynomials $J_{n}^{(\alpha, \beta)}$ which are normal for $\alpha, \beta \leq 0$ and $\rho$-normal for $\alpha, \beta<0$, with $\rho=\min \{-\alpha,-\beta\}$ [7]. Chebyshev nodes are a special case of $\frac{1}{2}$-normal nodes, since they are the zeros of the Chebyshev polynomials $J_{n}^{\left(-\frac{1}{2},-\frac{1}{2}\right)}$. For Chebyshev nodes, the upper bound in (6) can be improved significantly [10] to

$$
\Omega_{1, n} \leq \frac{\log n}{n} C,
$$

where $C$ is a constant independent of $n$. Other results about polynomial Hermite interpolation for normal and $\rho$-normal sets of nodes can be found in [11] and references therein.

However, this favourable behaviour does not hold in another common interpolation setting, the equidistant case with nodes

$$
x_{i}=\frac{i}{n}
$$

for $i=0, \ldots, n$. Indeed, both $\Omega_{0, n}$ and $\Omega_{1, n}$ grow very fast as $n \rightarrow \infty$, namely [6]

$$
\Omega_{0, n} \sim \frac{2^{2 n+1}}{\gamma_{n}^{2} n^{2}} C, \quad \Omega_{1, n} \sim \frac{2^{2 n+1}}{\gamma_{n}^{2} n^{2} \sqrt{n}} C,
$$

where $\gamma_{n}=\sum_{j=1}^{n} 1 / j$. This unfavourable growth also reflects the ill-conditioning of polynomial Hermite interpolation at equidistant nodes, which may lead to wild oscillations of $h_{n}$, just as in the Lagrange interpolation case.

In the Lagrange case, barycentric rational Floater-Hormann interpolants [4] have been shown to have a much better conditioning than polynomial interpolants at equidistant nodes, since the related Lebesgue constants grow only logarithmically with $n$ and exponentially with $d$, a parameter related to the construction and the approximation order of the Floater-Hormann interpolants [1,2]. In this paper we show that this favourable behaviour also holds for a generalization of these interpolants to the Hermite setting. After reviewing Cirillo and Hormann's [3] iterative construction of barycentric rational Hermite interpolants (Section 2), we show that for the special case of Floater-Hormann Hermite interpolation both $\Omega_{0, n}$ and $\Omega_{1, n}$ can be bounded from above by a constant that grows exponentially with $d$ (Section 3). We conclude with some numerical examples that confirm this result (Section 4).

\section{Iterative barycentric rational Hermite interpolation}

Schneider and Werner [8] show that the rational function

$$
r_{n}(x)=\sum_{i=0}^{n} b_{i}(x) f_{i},
$$

with basis functions

$$
b_{i}(x)=\frac{w_{i}}{x-x_{i}} / \sum_{k=0}^{n} \frac{w_{k}}{x-x_{k}}
$$


interpolates the data $f_{j}$ at $x_{j}$ for $j=0, \ldots, n$, as long as all the weights $w_{i}$ are non-zero. It is clear that these $b_{i}$ satisfy the Lagrange property

$$
b_{i}\left(x_{j}\right)=\delta_{i, j}
$$

and form a partition of unity,

$$
\sum_{i=0}^{n} b_{i}(x)=1
$$

just like the Lagrange basis polynomials $\ell_{i}$, and we recall from [8, Proposition 11] that

$$
b_{i}^{\prime}\left(x_{i}\right)=\sum_{\substack{j=0 \\ j \neq i}}^{n} \frac{w_{j}}{w_{i}\left(x_{j}-x_{i}\right)}, \quad b_{i}^{\prime}\left(x_{j}\right)=\frac{w_{i}}{w_{j}\left(x_{j}-x_{i}\right)}, \quad j \neq i .
$$

Cirillo and Hormann [3] show that these interpolants can be extended to the Hermite setting by letting

$$
h_{n}(x)=r_{n}(x)+q_{n}(x),
$$

where the correction term

$$
q_{n}(x)=\sum_{i=0}^{n}\left(x-x_{i}\right) b_{i}(x)^{2}\left(f_{i}^{\prime}-r_{n}^{\prime}\left(x_{i}\right)\right)
$$

is chosen so as to fix the interpolation of the derivative data $f_{i}^{\prime}$ without altering the interpolation of the data $f_{i}$. For our purposes, it turns out to be useful to rewrite the barycentric rational Hermite interpolant $h_{n}$ in (12) in the form (1) with $b_{0, i}$ and $b_{1, i}$ as in (2), but with $\ell_{i}$ replaced by $b_{i}$ in (8).

Proposition 2.1. The barycentric rational Hermite interpolant $h_{n}$ in (12) can be written as

$$
h_{n}(x)=\sum_{i=0}^{n} b_{0, i}(x) f_{i}+\sum_{i=0}^{n} b_{1, i}(x) f_{i}^{\prime}
$$

with

$$
b_{0, i}(x)=\left(1-2\left(x-x_{i}\right) b_{i}^{\prime}\left(x_{i}\right)\right) b_{i}(x)^{2}, \quad b_{1, i}(x)=\left(x-x_{i}\right) b_{i}(x)^{2} .
$$

Proof. By (12) and (7),

$$
h_{n}(x)=\sum_{i=0}^{n} b_{i}(x) f_{i}+\sum_{i=0}^{n}\left(x-x_{i}\right) b_{i}(x)^{2} f_{i}^{\prime}-\sum_{i=0}^{n}\left(x-x_{i}\right) b_{i}(x)^{2} \sum_{j=0}^{n} b_{j}^{\prime}\left(x_{i}\right) f_{j} .
$$

It remains to show that

$$
b_{i}(x)-\sum_{j=0}^{n}\left(x-x_{j}\right) b_{j}(x)^{2} b_{i}^{\prime}\left(x_{j}\right)=b_{i}(x)^{2}-2\left(x-x_{i}\right) b_{i}^{\prime}\left(x_{i}\right) b_{i}(x)^{2} .
$$

Using (10) and (11) we have

$$
\begin{aligned}
b_{i}(x)-\sum_{j=0}^{n}\left(x-x_{j}\right) b_{j}(x)^{2} b_{i}^{\prime}\left(x_{j}\right) & =b_{i}(x)^{2}+\sum_{\substack{j=0 \\
j \neq i}}^{n} b_{i}(x) b_{j}(x)-\left(x-x_{i}\right) b_{i}(x)^{2} b_{i}^{\prime}\left(x_{i}\right)-\sum_{\substack{j=0 \\
j \neq i}}^{n}\left(x-x_{j}\right) b_{j}(x)^{2} \frac{w_{i}}{w_{j}\left(x_{j}-x_{i}\right)} \\
& =b_{i}(x)^{2}-\left(x-x_{i}\right) b_{i}(x)^{2} b_{i}^{\prime}\left(x_{i}\right)+\sum_{\substack{j=0 \\
j \neq i}}^{n}\left(b_{i}(x)-\frac{x-x_{j}}{x_{j}-x_{i}} b_{j}(x) \frac{w_{i}}{w_{j}}\right) b_{j}(x) .
\end{aligned}
$$

Now, since

$$
b_{j}(x)=\frac{x-x_{i}}{x-x_{j}} b_{i}(x) \frac{w_{j}}{w_{i}}
$$

by (8), we find that

$$
\left(b_{i}(x)-\frac{x-x_{j}}{x_{j}-x_{i}} b_{j}(x) \frac{w_{i}}{w_{j}}\right) b_{j}(x)=\left(1-\frac{x-x_{i}}{x_{j}-x_{i}}\right) b_{i}(x) b_{j}(x)=-\left(x-x_{i}\right) b_{i}(x)^{2} \frac{w_{j}}{w_{i}\left(x_{j}-x_{i}\right)},
$$

and (14) then follows by using again (11). 


\section{Floater-Hormann Hermite interpolation}

Floater and Hormann [4] show that for any $d$ with $0 \leq d \leq n$, the special choice of barycentric weights

$$
w_{i}=(-1)^{i} v_{i}
$$

with

$$
v_{i}=\sum_{j=\max \{0, i-d\}}^{\min \{i, n-d\}} \prod_{\substack{k=j \\ k \neq i}}^{j+d} \frac{1}{\left|x_{i}-x_{k}\right|}>0
$$

in (8) gives barycentric rational interpolants without any real poles and approximation order $O\left(h^{d+1}\right)$ for sufficiently smooth functions. As shown in [2], in the special case of equidistant nodes, the corresponding Lebesgue constant grows logarithmically in the number of nodes. For $n \geq 2 d$ equidistant nodes, the values $v_{i}$ in (15) can be simplified to (cf. [2])

$$
v_{i}=\sum_{j=d}^{n}\left(\begin{array}{c}
d \\
j-i
\end{array}\right) \leq 2^{d},
$$

and we shall now derive an upper bound of the Lebesgue constant associated with the barycentric rational Hermite interpolant $h_{n}$ in (13) for this choice of $v_{i}$.

More precisely, we derive upper bounds for $\Omega_{0, n}$ and $\Omega_{1, n}$ in (4) and then use (3). Inspired by the proof of Theorem 1 in [2], we first focus on the case where $x_{k}<x<x_{k+1}$ for some $k$ with $0 \leq k \leq n-1$ and rewrite $\Omega_{0, n}(x)$ and $\Omega_{1, n}(x)$ as

$$
\Omega_{0, n}(x)=\frac{N_{0, k}(x)}{D_{k}(x)}, \quad \Omega_{1, n}(x)=\frac{N_{1, k}(x)}{D_{k}(x)},
$$

where

$$
\begin{aligned}
& N_{0, k}(x)=\left(x-x_{k}\right)^{2}\left(x_{k+1}-x\right)^{2} \sum_{i=0}^{n}\left|1-2\left(x-x_{i}\right) b_{i}^{\prime}\left(x_{i}\right)\right| \frac{v_{i}^{2}}{\left(x-x_{i}\right)^{2}}, \\
& N_{1, k}(x)=\left(x-x_{k}\right)^{2}\left(x_{k+1}-x\right)^{2} \sum_{i=0}^{n} \frac{v_{i}^{2}}{\left|x-x_{i}\right|},
\end{aligned}
$$

and

$$
D_{k}(x)=\left(x-x_{k}\right)^{2}\left(x_{k+1}-x\right)^{2}\left(\sum_{i=0}^{n} \frac{w_{i}}{x-x_{i}}\right)^{2} .
$$

As proved in [2], the denominator satisfies

$$
D_{k}(x) \geq \frac{1}{n^{2}},
$$

and it remains to establish appropriate upper bounds for the numerators $N_{0, k}(x)$ and $N_{1, k}(x)$.

Lemma 3.1. Let $n \geq 2 d$ and $x_{k}<x<x_{k+1}$ for some $k$ with $0 \leq k \leq n-1$. Then,

$$
N_{1, k}(x) \leq \frac{4^{d}}{n^{2}} C,
$$

for some constant $C$ that does not depend on $k, d$, and $n$.

Proof. Since

$$
\sum_{i=0}^{n} \frac{v_{i}^{2}}{\left|x-x_{i}\right|}=\sum_{i=0}^{k} \frac{v_{i}^{2}}{x-x_{i}}+\sum_{i=k+1}^{n} \frac{v_{i}^{2}}{x_{i}-x} \leq \sum_{i=0}^{k} \frac{v_{i}^{2}}{x-x_{k}}+\sum_{i=k+1}^{n} \frac{v_{i}^{2}}{x_{k+1}-x}
$$

and

$$
\left(x-x_{k}\right)\left(x_{k+1}-x\right)^{2} \leq \frac{4}{27 n^{3}}, \quad\left(x-x_{k}\right)^{2}\left(x_{k+1}-x\right) \leq \frac{4}{27 n^{3}},
$$

we have

$$
N_{1, k}(x) \leq \frac{4}{27 n^{3}} \sum_{i=0}^{n} v_{i}^{2}
$$

and the statement then follows from (16). 
Lemma 3.2. Let $n \geq 2 d$ and $x_{k}<x<x_{k+1}$ for some $k$ with $0 \leq k \leq n-1$. Then,

$$
N_{0, k}(x) \leq \frac{4^{d}(d+1)}{n^{2}} C,
$$

for some constant $C$ that does not depend on $k, d$, and $n$.

Proof. Using (11) and (16), we first notice that

$$
\sum_{i=0}^{n}\left|1-2\left(x-x_{i}\right) b_{i}^{\prime}\left(x_{i}\right)\right| \frac{v_{i}^{2}}{\left(x-x_{i}\right)^{2}} \leq 4^{d} \sum_{i=0}^{n} \frac{1}{\left(x-x_{i}\right)^{2}}+2^{d+1} \sum_{i=0}^{n} \frac{1}{\left|x-x_{i}\right|}\left|\sum_{\substack{j=0 \\ j \neq i}}^{n} \frac{(-1)^{j} v_{j}}{x_{j}-x_{i}}\right|
$$

and we proceed to bound the two sums over $i$ separately. For $x_{k}<x<x_{k+1}$, we have

$$
\begin{aligned}
\sum_{i=0}^{n} \frac{1}{\left(x-x_{i}\right)^{2}} & =\sum_{i=0}^{k-1} \frac{1}{\left(x-x_{i}\right)^{2}}+\frac{1}{\left(x-x_{k}\right)^{2}}+\frac{1}{\left(x_{k+1}-x\right)^{2}}+\sum_{i=k+2}^{n} \frac{1}{\left(x_{i}-x\right)^{2}} \\
& \leq \sum_{i=0}^{k-1} \frac{1}{\left(x_{k}-x_{i}\right)^{2}}+\frac{\left(x_{k+1}-x_{k}\right)^{2}}{\left(x-x_{k}\right)^{2}\left(x_{k+1}-x\right)^{2}}+\sum_{i=k+2}^{n} \frac{1}{\left(x_{i}-x_{k+1}\right)^{2}} \\
& =\sum_{i=0}^{k-1} \frac{n^{2}}{(k-i)^{2}}+\frac{1}{n^{2}} \frac{1}{\left(x-x_{k}\right)^{2}\left(x_{k+1}-x\right)^{2}}+\sum_{i=k+2}^{n} \frac{n^{2}}{(i-k-1)^{2}} \\
& =n^{2} \sum_{i=1}^{k} \frac{1}{i^{2}}+\frac{1}{n^{2}} \frac{1}{\left(x-x_{k}\right)^{2}\left(x_{k+1}-x\right)^{2}}+n^{2} \sum_{i=1}^{n-k-1} \frac{1}{i^{2}} \\
& \leq n^{2} \frac{\pi^{2}}{6}+\frac{1}{n^{2}} \frac{1}{\left(x-x_{k}\right)^{2}\left(x_{k+1}-x\right)^{2}}+n^{2} \frac{\pi^{2}}{6},
\end{aligned}
$$

and since

we conclude that

$$
\left(x-x_{k}\right)^{2}\left(x_{k+1}-x\right)^{2} \leq \frac{1}{16 n^{4}},
$$

$$
\left(x-x_{k}\right)^{2}\left(x_{k+1}-x\right)^{2} \sum_{i=0}^{n} \frac{1}{\left(x-x_{i}\right)^{2}} \leq \frac{1}{n^{2}} C .
$$

To bound the second sum, we first use (16) to get

$$
\left|\sum_{\substack{j=0 \\
j \neq i}}^{n} \frac{(-1)^{j} v_{j}}{j-i}\right|=\left|\sum_{\substack{j=0 \\
j \neq i}}^{n} \frac{(-1)^{j}}{j-i} \sum_{l=d}^{n}\left(\begin{array}{c}
d \\
l-j
\end{array}\right)\right|=\left|\sum_{l=0}^{d}\left(\begin{array}{c}
d \\
l
\end{array}\right) \sum_{\substack{j=d-l \\
j \neq i}}^{n-l} \frac{(-1)^{j}}{j-i}\right| \leq 2^{d} \max _{0 \leq l \leq d}\left|\sum_{\substack{j=d-l \\
j \neq i}}^{n-l} \frac{(-1)^{j}}{j-i}\right|,
$$

and since

$$
\begin{aligned}
& \left|\sum_{j=d-l}^{n-l} \frac{(-1)^{j}}{j-i}\right| \leq \frac{1}{(d-l)-i}, \quad 0 \leq i<d-l, \\
& \left|\sum_{\substack{j=d-l \\
j \neq i}}^{n-l} \frac{(-1)^{j}}{j-i}\right|=\left\{\left|\sum_{j=1}^{i-(d-l)} \frac{(-1)^{j}}{j}-\sum_{j=1}^{(n-l)-i} \frac{(-1)^{j}}{j}\right| \leq \begin{cases}\frac{1}{i-(d-l)+1}, & d-l \leq i \leq \frac{n+d}{2}-l, \\
\frac{1}{(n-l)-i+1}, & \frac{n+d}{2}-l \leq i \leq n-l,\end{cases} \right. \\
& \left|\sum_{j=d-l}^{n-l} \frac{(-1)^{j}}{i-j}\right| \leq \frac{1}{i-(n-l)}, \quad n-l<i \leq n,
\end{aligned}
$$

we further have

$$
c_{i}=\left|\sum_{\substack{j=0 \\ j \neq i}}^{n} \frac{(-1)^{j} v_{j}}{j-i}\right| \leq 2^{d} \begin{cases}1, & 0 \leq i \leq d, \\ \frac{1}{i-d+1}, & d \leq i \leq \frac{n}{2}, \\ \frac{1}{(n-d)-i+1}, & \frac{n}{2} \leq i \leq n-d, \\ 1, & n-d \leq i \leq n .\end{cases}
$$


Let us now assume that $d \leq k<n / 2-1$ and $x_{k}<x<x_{k+1}$. Then,

$$
\sum_{i=0}^{k-1} \frac{c_{i}}{k-i} \leq 2^{d}\left(\sum_{i=0}^{d-1} \frac{1}{k-i}+\sum_{i=d}^{k-1} \frac{1}{(k-i)(i-d+1)}\right) \leq 2^{d}(d+1),
$$

and

$$
\frac{c_{k}}{x-x_{k}} \leq \frac{2^{d}}{x-x_{k}}, \quad \frac{c_{k+1}}{x_{k+1}-x} \leq \frac{2^{d}}{x_{k+1}-x},
$$

and

$$
\begin{aligned}
\sum_{i=k+2}^{n} \frac{c_{i}}{i-k-1} & \leq 2^{d}\left(\sum_{i=k+2}^{\lfloor n / 2\rfloor} \frac{1}{i-k-1}\left(\frac{1}{i-d+1}-\frac{1}{n-d-i+1}\right)\right. \\
& \left.+\sum_{i=k+2}^{n-d} \frac{1}{(i-k-1)(n-d-i+1)}+\sum_{i=n-d+1}^{n} \frac{1}{i-k-1}\right) \\
& \leq 2^{d}\left(\sum_{i=k+2}^{\lfloor n / 2\rfloor} \frac{1}{(i-k-1)(i-d+1)}+\sum_{i=1}^{n-d-k-1} \frac{1}{i(n-d-k-i)}+\sum_{i=n-d}^{n-1} \frac{1}{i-k}\right) \\
& \leq 2^{d}\left(\frac{\pi^{2}}{6}+1+d\right) .
\end{aligned}
$$

Therefore,

$$
\begin{aligned}
\sum_{i=0}^{n} \frac{1}{\left|x-x_{i}\right|}\left|\sum_{\substack{j=0 \\
j \neq i}}^{n} \frac{(-1)^{j} v_{j}}{x_{j}-x_{i}}\right| & \leq n^{2} \sum_{i=0}^{k-1} \frac{c_{i}}{k-i}+n \frac{c_{k}}{x-x_{k}}+n \frac{c_{k+1}}{x_{k+1}-x}+n^{2} \sum_{i=k+2}^{n} \frac{c_{i}}{i-k-1} \\
& \leq 2^{d}\left(n^{2}(d+1)+\frac{n}{x-x_{k}}+\frac{n}{x_{k+1}-x}+n^{2}\left(\frac{\pi^{2}}{6}+1+d\right)\right) .
\end{aligned}
$$

Using (20) and (21), we finally obtain

$$
\left(x-x_{k}\right)^{2}\left(x_{k+1}-x\right)^{2} \sum_{i=0}^{n} \frac{1}{\left|x-x_{i}\right|}\left|\sum_{\substack{j=0 \\ j \neq i}}^{n} \frac{(-1)^{j} v_{j}}{x_{j}-x_{i}}\right| \leq \frac{2^{d}(d+1)}{n^{2}} C .
$$

The other cases $k<d$ and $k \geq n / 2-1$ can be treated similarly.

We are now ready to state our main result.

Theorem 3.3. Provided that $n \geq 2 d$, the Lebesgue constant associated with Floater-Hormann Hermite interpolation at equidistant nodes satisfies

$$
\Lambda_{n} \leq 4^{d}(d+1) C,
$$

for some constant $C$ that does not depend ond and $n$.

Proof. If $x=x_{k}$ for $k=0, \ldots, n$, then

$$
b_{0, i}(x)=\left(1-2\left(x_{k}-x_{i}\right) b_{i}^{\prime}\left(x_{i}\right)\right) b_{i}\left(x_{k}\right)^{2}=\delta_{i, k}, \quad b_{1, i}(x)=\left(x_{k}-x_{i}\right) b_{i}\left(x_{k}\right)^{2}=0
$$

and consequently $\Omega_{0, n}(x)=1$ and $\Omega_{1, n}(x)=0$. Otherwise, it follows from (19), Lemma 3.1, and Lemma 3.2, that there exists some constant $C$ that does not depend on $n$ and $d$, such that

$$
\Omega_{0, n}(x) \leq 4^{d}(d+1) C, \quad \Omega_{1, n}(x) \leq 4^{d} C .
$$

The statement then follows from (3).

Note that while the constant $C$ in Theorem 3.3 is independent of $d$, the upper bound grows exponentially in $d$. 

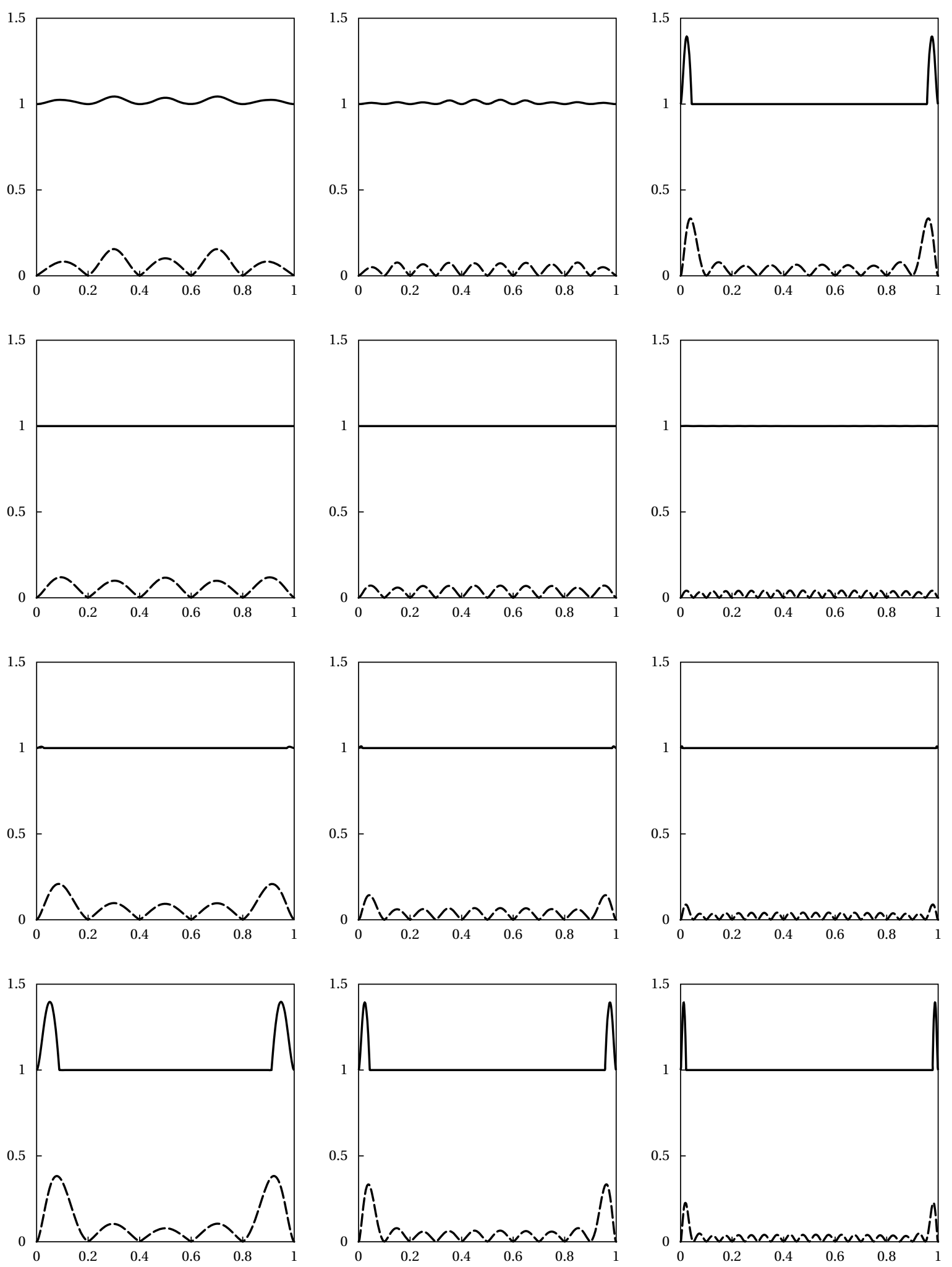

Figure 1: Plot of $\Omega_{0, n}(x)$ (solid line) and $\Omega_{1, n}(x)$ (dashed line) for $d=0,1,2,3$ (from top to bottom) and $n=5,10,20$ (from left to right) and equidistant nodes in the interval $[0,1]$. 

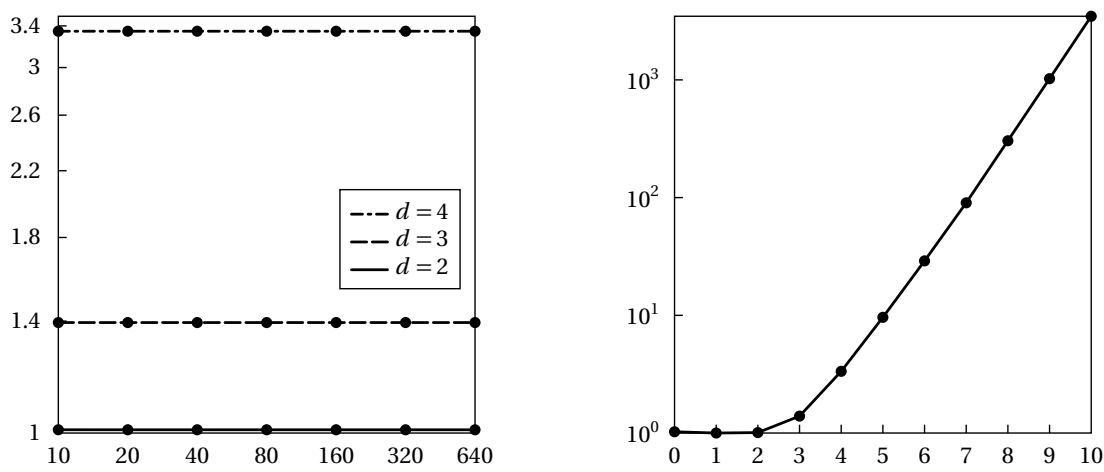

Figure 2: Log-log plot of $\Omega_{0, n}$ over $n$ for different values of $d$ (left) and semi-log plot of $\Omega_{0, n}$ over $d$ for $n=20$ (right).

\section{Numerical results}

We performed several experiments to confirm numerically that the upper bounds derived above are correct. Figure 1 shows $\Omega_{0, n}(x)$ and $\Omega_{1, n}(x)$ for Floater-Hormann Hermite interpolation at equidistant nodes in the interval $[0,1]$ for several values of $d$ and $n$. Note that $\Omega_{0, n}(x)$ dominates $\Omega_{1, n}(x)$ in all examples, a behaviour that we consistently observed in our experiments. Also note that the maxima $\Omega_{0, n}$ and $\Omega_{1, n}$ of both functions are obtained inside the first and the last sub-interval, except for $d=0$, and that $\Omega_{0, n}$ is basically independent of $n$ in all examples. This is confirmed by the plot in Figure 2 (left), which additionally shows that $\Omega_{0, n}$, although independent of $n$, seems to grow exponentially with $d$, as suggested by the upper bound in Lemma 3.2. This trend can also be observed in Figure 2 (right), where the same quantity is plotted for a fixed value of $n$ and $d$ between 0 and $n / 2$.

A completely different result can be observed for non-equidistant nodes. For example, in the case of Chebyshev nodes, $\Omega_{0, n}$ grows quickly as $n$ increases, except for $d=0$, as shown in Figure 3 . We therefore recommend to use Floater-Hormann Hermite interpolation for equidistant nodes, but to stick to polynomial Hermite interpolants for Chebyshev nodes. It remains future work to investigate other choices of interpolation nodes.

\section{Acknowledgements}

This work was supported by the Swiss National Science Foundation (SNSF) under project number 200021_150053.

\section{References}

[1] L. Bos, S. De Marchi, and K. Hormann. On the Lebesgue constant of Berrut's rational interpolant at equidistant nodes. Journal of Computational and Applied Mathematics, 236(4):504-510, Sept. 2011.

[2] L. Bos, S. De Marchi, K. Hormann, and G. Klein. On the Lebesgue constant of barycentric rational interpolation at equidistant nodes. Numerische Mathematik, 121(3):461-471, July 2012.

[3] E. Cirillo and K. Hormann. An iterative approach to barycentric rational Hermite interpolation. Numerische Mathematik, 140(4):939-962, Dec. 2018.

[4] M. S. Floater and K. Hormann. Barycentric rational interpolation with no poles and high rates of approximation. Numerische Mathematik, 107(2):315-331, Aug. 2007.

[5] C. Hermite. Sur la formule d'interpolation de Lagrange. Journal für die reine und angewandte Mathematik, 84:70-79, 1878.

[6] C. Manni. Lebesgue constants for Hermite and Fejér interpolation on equidistant nodes. Calcolo, 30(3):203-218, Sept. 1993.

[7] I. P. Natanson. Constructive Function Theory, volume III. Frederick Ungar, New York, 1965.

[8] C. Schneider and W. Werner. Some new aspects of rational interpolation. Mathematics of Computation, 47(175):285299, July 1986.

[9] J. Stoer and R. Bulirsch. Introduction to Numerical Analysis, volume 12 of Texts in Applied Mathematics. SpringerVerlag, New York, 2nd edition, 1993.

[10] J. Szabados. On the order of magnitude of fundamental polynomials of Hermite interpolation. Acta Mathematica Hungarica, 61(3-4):357-368, Sept. 1993. 

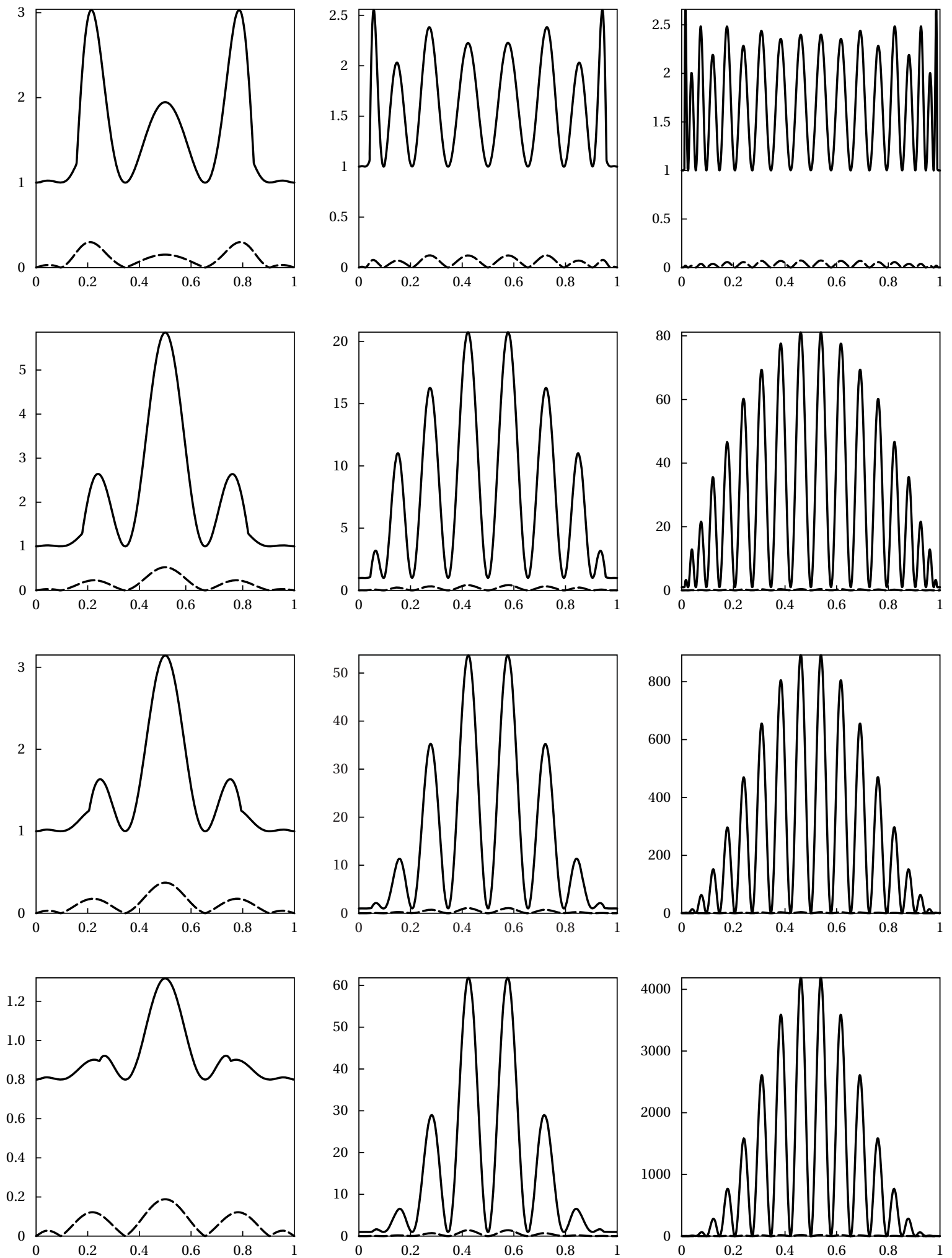

Figure 3: Plot of $\Omega_{0, n}(x)$ (solid line) and $\Omega_{1, n}(x)$ (dashed line) for $d=0,1,2,3$ (from top to bottom) and $n=5,10,20$ (from left to right) and Chebyshev nodes in the interval $[0,1]$.

[11] P. Vértesi. Recent results on Hermite-Fejér interpolation of higher order (uniform metric). In S. Baron and D. Leviatan, editors, Approximation, Interpolation, and Summability, volume 4 of Israel Mathematical Conference Proceedings, pages 267-271. American Mathematical Society, Providence, 1991. 